\title{
Overview and Prospect of distributed energy storage technology
}

\author{
Peng Ye ${ }^{1, *}$, Siqi Liu ${ }^{1}$, Feng Sun ${ }^{2}$, Mingli Zhang ${ }^{3}$, and Na Zhang ${ }^{3}$ \\ ${ }^{1}$ Shenyang Institute of engineering, Shenyang 110136, China \\ ${ }^{2}$ State Grid Liaoning Electric Power Supply Co.LTD, Electric Power Research Insitute, Shenyang 110006, China \\ ${ }^{3}$ State Grid Liaoning Electric Power Company Limited Economic Research Institute, Shenyang, 110065, China
}

\begin{abstract}
The combination of distributed generation and distributed energy storage technology has become a mainstream operation mode to ensure reliable power supply when distributed generation is connected to the grid. This paper first introduces two typical distributed energy storage technologies: pumped storage and battery energy storage. Then, it introduces the energy storage technologies represented by the "ubiquitous power Internet of things" in the new stage of power industry, such as virtual power plant, smart micro grid and electric vehicle. Finally, this paper summarizes and prospects the distributed energy storage technology.
\end{abstract}

\section{Introduction}

Based on the strategy of sustainable development and reducing the dependence on traditional fossil energy in energy consumption, the national development and Reform Commission issued the 12th Five Year Plan for renewable energy development in 2012, in which the policy of "promoting energy efficiency and cleaning, and reform of supply mode" is specified in detail. In recent years, with the promotion of this policy and the state subsidies to the new energy industry, new energy projects have sprung up everywhere. In 2017, the newly added installed capacity of photovoltaic power generation reached $52.83 \mathrm{GW}$, which is the highest point in history by the end of 2019. Compared with the national subsidy standard, nearly $35 \%$ of the newly added installed capacity is over capacity every year. Moreover, in the process of large-scale grid connection of new energy power generation, the intermittence, randomness and volatility of power generation will reduce the power quality and reliability of power supply. From 2018, the state will reduce the subsidies to the new energy industry, and is expected to shift the focus of subsidies to distributed energy storage technology and power grid stability.

Distributed energy storage has small power and capacity, and its access location is flexible. It is usually concentrated in the user side, distributed microgrid and medium and low voltage distribution network. It can be used for peak load regulation, frequency regulation, and improving the power quality and reliability of power supply. Distributed energy storage can be divided into mechanical energy storage, electromagnetic energy storage (physical energy storage), battery energy storage and hydrogen energy storage (chemical energy storage). The common types are: pumped storage power station, flywheel energy storage, compressed air energy storage (mechanical energy storage); superconducting, super capacitor energy storage (electromagnetic energy storage); electrochemical energy storage, electric vehicles, etc. Among them, pumped storage power stations are widely used, accounting for about $90 \%$ of the total installed capacity of various types of distributed energy storage. Most of its installed capacity is concentrated in North China, East China and central China, and Northeast China is also developing vigorously. Electrochemical energy storage is a more concentrated research direction at present. Lithium batteries and lead-acid batteries occupy most of the electronic market share. The research and development cost of electromagnetic energy storage is high, and its application is less at present. In the environment of micro grid system and distributed generation of renewable energy, distributed energy storage, as an effective technology to improve the power quality after grid connection, is bound to become the next development trend. Under the background of distributed energy storage marketization, it is necessary to study its market operation mechanism, how to cooperate with power grid operation and network operation platform architecture.

Reference [1] puts forward an operation mode beneficial to the profit of distributed energy storage project based on the "leverage effect" in economics. Reference [2] establishes different interactive package systems and carries out risk assessment for three operation mechanisms, which provides a reference for the operation of customer side energy storage market. In reference [3], the rationality of the market incentive mechanism is analyzed through the incentive effect evaluation model of fuzzy clustering in the bidding stage of power generation. Although the energy storage market is not mentioned, the results are also applicable. Reference [4] takes a specific family as an example to analyze the cost and benefit of putting photovoltaic power generation system into operation. In reference [5], 
the fuzzy clustering method of improved Mahalanobis distance is used to classify the users with different responses into five categories under the environment of electricity price incentive. It is pointed out that with the development of distributed generation access and energy storage technology, the power consumption of users will be more diverse and uncertain, and the research on the operation mechanism of distributed energy storage is the main direction in the future.

In this paper, from the two aspects of distributed energy storage and its market operation mechanism, we summarize the battery energy storage and pumped storage technologies which cover a wide range at present, and then from the new stage of "ubiquitous power Internet of things" in the power industry, we summarize the new distributed energy storage technologies such as virtual power plant, smart microgrid and electric vehicle. Finally, this paper summarizes and prospects the distributed energy storage technology.

\section{Distributed energy storage technology}

\subsection{Pumped storage}

Pumped storage accounts for the majority of the energy storage market in China. Such as Beijing Ming Tombs, Guangzhou phase I phase II, Shandong Tai-an, Jiangsu Yi-xing and other storage power stations. By 2020, the operation capacity of pumped storage in China is expected to reach $40 \mathrm{GW}$. The principle of pumped storage is: in the low electricity consumption or wet season, the abundant electric energy is converted into mechanical energy, and the water in the lower reservoir is moved to the upper reservoir; in the peak electricity consumption or dry season, the stored water is released, and the potential energy in the fall is converted into mechanical energy, so as to drive the turbine to generate electricity. Its main functions of auxiliary operation in power grid are as follows:

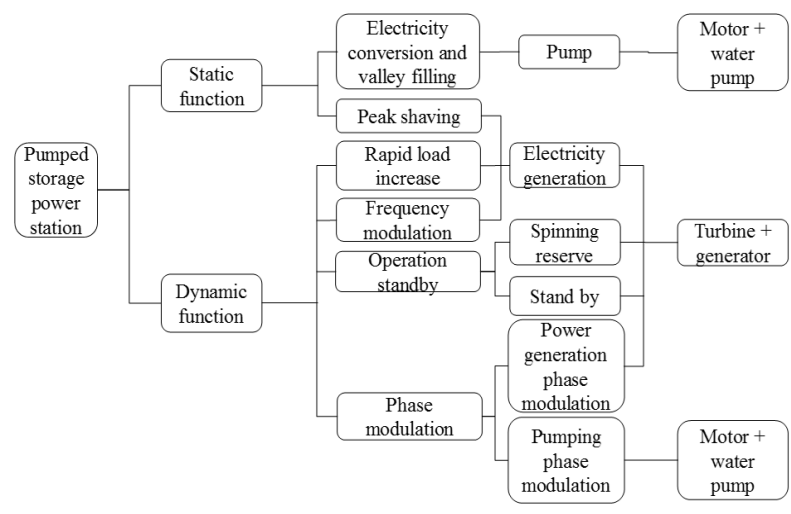

Figure 1. Main functions of pumped storage in auxiliary operation of power grid

Pumped storage is a relatively mature energy storage technology in China. In 2014, the state uniformly implemented the "two-part" electricity price for pumped storage power stations. The "two parts" refer to the capacity price and the electricity price. The capacity price is a fixed part, which is the cost paid by the power grid to the fixed equipment capacity of the pumped storage power station. It is mainly based on the auxiliary operation of the pumped storage power station to the power grid, so as to make up for the fixed cost and permitted income of the power station. Electricity price is a variable cost, which is the cost of purchasing electricity from pumped storage power station. The price of electricity will produce a variable cost, which is mainly based on the variable income of electricity market transaction, to make up for the variable cost of power generation loss. The main operation modes of pumped storage power stations are: (1) unified operation of power grid; (2) multi-party joint leasing; (3) independent operation; (4) entrusted operation of power grid. For example, Beijing Ming Tombs power station adopts independent operation mode in the initial stage, and Guangzhou Pumped Storage Power Station adopts leasing mode in the later stage. The operation mode of power station is not single. With the change of the whole power market and its own operation benefit, it will also change.

Capacity price and electricity price are as follows:

$$
\begin{aligned}
& R_{\text {capacity }}=k \lambda_{c}\left(C_{P}+C_{R}-C_{A}\right) \\
& R_{\text {energy }}=k \lambda_{q} Q_{G}
\end{aligned}
$$

In the above formula, $k$ is the product of the unit availability adjustment coefficient and the power market supply and demand coefficient, $\lambda_{c}$ is the capacity price, $C_{P}$ is the annual planned available capacity, $C_{R}$ is the reserve capacity, $C_{A}$ is the adjusted capacity, $\lambda_{q}$ is the electricity price, and $Q_{G}$ is the on grid power generation.

\subsection{Battery energy storage}

Battery energy storage is a device that converts chemical energy and electric energy into each other based on the redox reaction on the electrode side. Unlike some fixed large-scale energy storage power stations, battery energy storage can be used as both fixed energy storage devices and mobile energy storage facilities, so in some mobile tools such as electric vehicles, energy storage batteries are indispensable. On the other hand, battery energy storage is a DC power supply equipment, which can ensure the reliability of power supply quality. Therefore, in all kinds of distributed generation technologies, such as wind power, "back-to-back system" in photovoltaic power generation, BMS and PCS system in intelligent microgrid, lithium-ion battery or lead-acid battery are used to realize flexible adjustment, flat sliding, ensuring power index stability and other functions.

Vanadium redox flow battery is also a kind of energy storage battery which is widely used at present. Its electrode material is flow redox reaction electrode pair, such as zinc bromide battery and zinc chloride battery. Compared with other batteries, alum flow battery has the following advantages: (1) single reactant between positive and negative electrodes, no cross contamination 
between positive and negative materials; (2) flexible control of energy storage capacity and SOC by increasing the volume of electrolyte, good internal uniformity of battery, no easy explosion hazard; (3) stack as the core component (4) long cycle life and excellent charge discharge performance; (5) environmentally friendly materials have become a new generation of clean energy storage batteries. In 2013, Liaoning invested and built a 5MW all vanadium flow battery energy storage power station in Wo-Niu-Shi, becoming the largest power station with all vanadium flow as energy storage mode. The hybrid model of flow cell and super-capacitor is as follows ${ }^{[6]}$ :

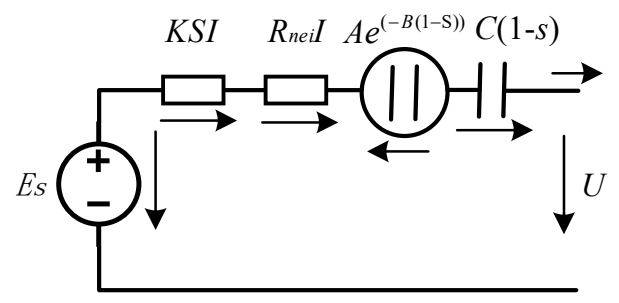

Figure 2. C.M.Shegherd model of flow cell

Among them, KSI is the voltage drop of electrode plate, $R_{n e i} I$ is the internal resistance, $A e^{(-B(1-S))}$ is the voltage drop at the beginning of discharge, and $C(1-s)$ is the no-load voltage correction.

In addition, some new energy storage batteries, such as nickel zinc battery, lithium sulfur battery, sodium ion battery and magnesium battery, have been developed one after another, which is expected to become the next "successor" of energy storage batteries.

Taking the benefit brought by part of auxiliary operation of energy storage battery market as an example, the profit mechanism can be expressed as follows:

(1) According to the time of use electricity price difference, the profit of "high storage and low generation" is as follows:

$$
R_{\text {gap }}=\sum_{d a y=1}^{365} \sum_{i=1}^{n} \lambda_{\text {gap }} P_{i} \square t_{i} \eta
$$

Among them, $R_{g a p}$ is the profit (yuan) obtained by profit model (1) $n$ is the number of time periods divided every day, $\square t_{i}$ is the interval of time periods, $\lambda_{\text {gap }}$ is the price difference between charging and discharging in the first time period, $P_{i}$ is the battery charging and discharging power in each time period, $\eta$ is the battery charging and discharging efficiency.

(2) By reducing the replacement cost and ensuring the compensation and subsidy profit of high-quality power supply:

$$
R_{\text {subsidy }}=\xi \eta P_{\max } C+T_{\text {sub }}
$$

Among them, $R_{\text {subsidy }}$ is the profit (yuan) obtained by profit model (2). $\xi$ is the depreciation rate of the original equipment, $\eta$ is the efficiency of the newly introduced energy storage battery, $P_{\max }$ is the rated power of the newly introduced energy storage battery, $C$ is the unit capacity cost of the original equipment (10000 yuan, $\mathrm{MW})$, and $T_{\text {sub }}$ is the subsidy.

(3) By supporting the stable demand response between the "grid-user" of distributed generation, the profit can be achieved as follows:

$$
R_{\text {storage }}=\lambda \sum_{k=1}^{365} \int_{t_{1}}^{t_{2}} p_{e}(t) d t
$$

Among them, $R_{\text {storage }}$ is the profit (yuan) obtained by profit model (3). The upper and lower limits $t_{s}$ represent the starting and ending periods when the phenomenon of "wind abandonment" or "light abandonment" occurs, $p_{e}(t)$ represents the power stored by the battery during the period of "wind abandonment" or "light abandonment" at time $t$, the integral formula represents the annual wind power or photovoltaic capacity (MWh) received more after the introduction of the energy storage battery, and $\lambda_{e}$ is the grid price of wind power or photovoltaic power.

\subsection{New form of energy storage}

With the change of the power grid structure and the updating of interaction with users, the concept of "Internet plus" and power ubiquitous Internet of things has become a new research direction. Smart microgrid, virtual power plant and electric vehicle can be the representative of energy storage operation mechanism in the new situation.

(1) Smart microgrid: smart microgrid has been implemented in China for a period of time, and its advantages are that it can form a network independently and operate in grid. However, due to its decentralized and independent, it will lead to the phenomenon of "island effect" or voltage sag when the auxiliary machine starts. The intervention of distributed energy storage equipment can effectively alleviate these unstable factors. At the same time, because of its intervention, the smart microgrid can also be regarded as a distributed energy storage system. Distributed energy storage facilities in microgrid are often used in AC / DC hybrid distribution system as "back-to-back system". After wind turbine and photovoltaic power generation, $\mathrm{AC}$ is converted into DC through "back-to-back system", and charging and discharging behavior is completed in energy storage battery. 


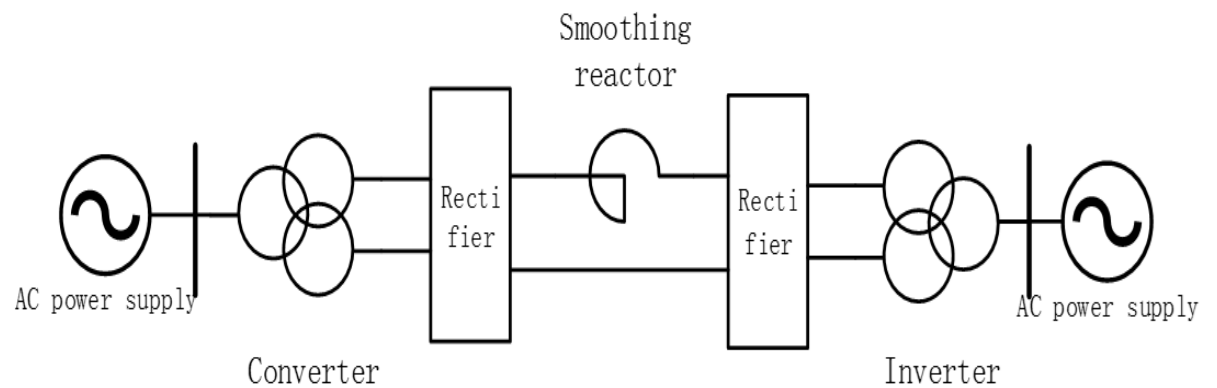

Figure 3. "back to back system" in wind turbine and photovoltaic power generation

For example, based on droop control combined with outer loop power control, the output power of distributed energy storage can be smoothly controlled.

(2) Virtual power plant: virtual power plant is a concept that integrates all kinds of distributed energy and participates in the electricity market transaction in the form of "virtual power plant" with auxiliary services through optimization and regulation. Virtual power plant is based on the concept of power ubiquitous Internet of things, because power ubiquitous Internet of things is an effective terminal network to realize data flow convergence "cloud computing". The regulation and integration of resources of virtual power plant cannot be separated from the dependence on data. It can be said that the virtual power plant is very similar to the energy storage cloud platform built in most areas, whose purpose is to aggregate all kinds of resources, assist power grid operation, and optimize power quality. The operation mechanism of the virtual power plant is as follows:

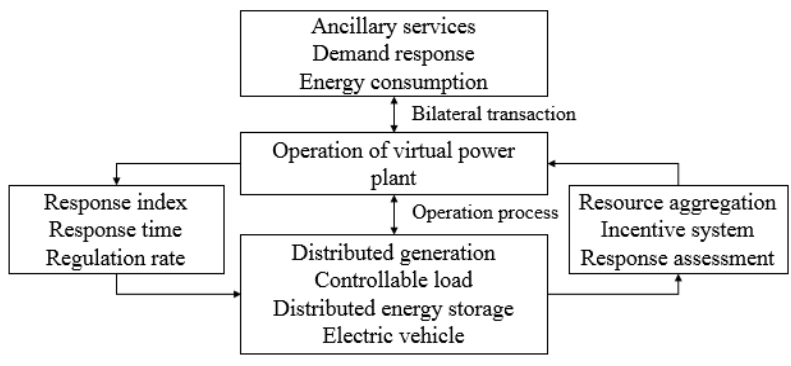

Figure 4. Operation mechanism of virtual power plant

At present, the common virtual power plant is the combination of distributed generation and energy storage, such as "electric vehicle building energy storage" system. The profit model of virtual power plant is similar to that of energy storage facilities. It can make profits through auxiliary services and energy consumption. It still needs policy promotion and further development.

(3) Electric vehicles:

The behavior of charging and discharging energy between electric vehicle and power grid can also be regarded as a distributed energy storage technology. At present, with the promotion of electric vehicles and the relaxation of subsidy policies, electric vehicles can be regarded as a group of mobile energy storage devices.
Reference [4] has set up a "plug and play" device as the medium between the access layer of electric vehicles and the energy storage management platform, which is composed of BMS management system, energy storage inverter, etc. The battery model commonly used in electric vehicle BMS management system is PNGV model. Taking lead-acid battery as an example, its input end is current, initial SOC and rated capacity, and its output end is terminal voltage and SOC, as shown in the figure below:

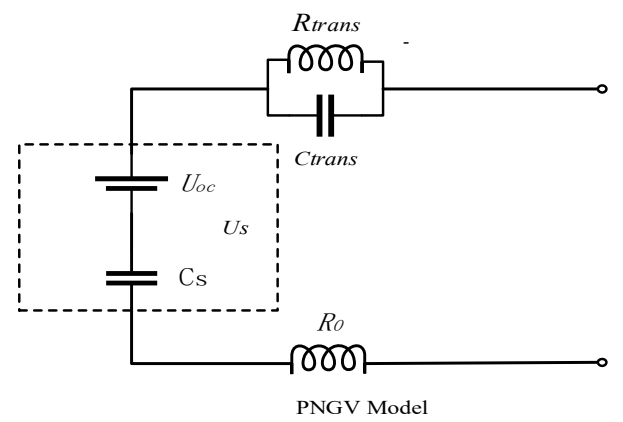

Figure 5. PNGV battery model of electric vehicle BMS management system

\section{Conclusion}

To sum up, the new technologies represented by smart microgrid, virtual power plant and electric vehicle, which not only contain power generation mechanism, but also can be used as energy storage facilities, bring about the change of energy structure and the update of interaction mode between grid and users, and more and more power market mechanisms emerge as the times require.

\section{References}

1. Zhao Wei, Xiao Xiang, Zheng Yaodong, et al. Network Operation Platform Architecture and Control Strategy of Distributed Energy Storage network Systems [J]. High voltage technology, 2019

2. Shi Zhiyong, Hong Bowen, Huang bibin, et al. Research on distributed energy storage operation mode and application value of in distribution network [J]. Power Demand Side Management, $2019(5)$ 
3. Chen Haisheng, Liu Chang, Qi Zhiping. Development trend and Present Status of Distributed Energy Storage [J]. Bulletin of Chinese Academy of Sciences, 2016, v.31 (02): 74-81

4. Yan Tao, Liu Zong, Ba Yu, Fei Juntao, Chen Jizhong, $\mathrm{Xu}$ Shaohua, Niu Meng. Research on distributed energy storage controller and control strategy based on Energy Storage Cloud Platform [J]. Electrical \& Energy Management Technology, 2019, no.563,59-64 + 71

5. Liu Jingkun, Zhang Ning, Kang Chongqing. Research Framework and Basic Models for Cloud Energy Storage in Power System[J]. Proceedings of The Chinese Society for Electrical Engineering, 037(12):3361-3371.

6. Bi Wei. Research on application of distributed energy storage system technology based on energy cloud management platform [J]. Electrical Engineering, 2018, 19 (4): 67-71 